\title{
Rational energy decay rate for a wave equation with dynamical control
}

\author{
Ali Wehbe*
}

\begin{abstract}
We consider a wave equation with dynamical control. We first establish the rational energy decay rate using a multiplier method. Next, using a spectrum method, we prove that the rational energy decay rate is optimal.
\end{abstract}

\section{Introduction}

In this work, we study the stabilization of one-dimensional wave equation with a dynamical control

$$
\begin{cases}y_{t t}-y_{x x}=0, & 0<x<1, \\ y(0, t)=0 & \\ y_{x}(1, t)+\eta(t)=0 & \\ \eta_{t}(t)-y_{t}(1, t)+\beta \eta(t)=0 & \end{cases}
$$

where $\eta$ designates the dynamical control and $\beta$ is a positive constant. The dynamical control has been introduced by the automaticians in the finite dimensional case (ordinary differential equations) see Francis [4], for example. In the infinite dimensional case, this concept of dynamical control is very close to the one of indirect damping mechanisms proposed by Russell [15].

Let $V=\left\{y \in H^{1}(0,1): y(0)=0\right\}$. We define the energy space $\mathcal{H}=V \times$ $L^{2}(0,1) \times \mathbb{R}$, endowed with the inner product:

$$
(u, \widetilde{u})_{\mathcal{H}}=\int_{0}^{1} y_{x} \widetilde{y}_{x} d x+\int_{0}^{1} z \widetilde{z} d x+\eta \widetilde{\eta}, \quad u=(y, z, \eta), \widetilde{u}=(\widetilde{y}, \widetilde{z}, \widetilde{\eta}) \in \mathcal{H} .
$$

Article published by EDP Sciences and available at http://www.edpsciences.org/proc or http://dx.doi.org/10.1051/proc:2000012

Next we define the linear operators $A$ and $B$ by

$$
\begin{gathered}
D(A)=\left\{u=(y, z, \eta) \in \mathcal{H}: y \in H^{2}(0,1), z \in V \text { and } y_{x}(1)+\eta=0\right\}, \\
A u=\left(z, y_{x x}, z(1)\right), \quad \forall u=(y, z, \eta) \in D(A),
\end{gathered}
$$

\footnotetext{
*Institut de Recherche Mathématique Avancée, Université Louis Pasteur de Strasbourg, 7, rue René-Descartes, 67084 Strasbourg Cedex, France. e-mail: wehbe@math.u-strasbg.fr
} 


$$
B u=(0,0,-\beta \eta), \quad \forall u=(y, z, \eta) \in \mathcal{H}
$$

Setting $u=\left(y, y_{t}, \eta\right) \in D(A)$, we formally transform the equation (1.1) into an evolutionary equation

$$
u_{t}=(A+B) u, \quad u(0)=u_{0} \in \mathcal{H} .
$$

It is easy to prove that $A$ is m-dissipative and $B$ is dissipative operators, then $A+B$ generates a $C_{0}$ semigroup $S(t)$ of contractions on the energy space $\mathcal{H}$ (see Pazy [10]). Moreover, since $A$ is skew adjoint and $B$ is compact, then using the compact perturbation theory of Russell [14], the system (1.1) is not uniformly stable (see Rao [12]). However, using the spectral decomposition theory of Sz-Nagy-Foias and Foguel (see Benchimol [1]), we can prove that the energy $E(t)$ decreases asymptotically to zero: $\lim _{t \rightarrow+\infty} E(t)=0, \quad \forall u_{0} \in \mathcal{H}$. It is well-known that the energy has not a preassigned decay rate. In fact, Littman and Markus in [9] proved that for any given decay rate there is an initial data $u_{0} \in \mathcal{H}$ such that the decay rate of the associated energy is less than the given one.

In this paper, our main concern is the energy decay rate for smooth solution. We will prove that for any $u_{0} \in D(A)$ the energy of the system (1.1) has a rational decay rate:

$$
E(t) \leq E(0) \frac{2 M}{M+t}, \quad \forall t \geq 0
$$

where $\mathrm{M}$ is a positive constant dependent on $u_{0}$. To this end, we employ a nonlinear technique used in Rao [11] for the dynamic control of the Kirchhoff plate. We next prove that the rational energy decay rate (1.3) is optimal in the sense that for any $\varepsilon>0$ there exists $u_{0}^{\varepsilon} \in D(A)$ such that the associated energy satisfies the estimate:

$$
E^{\varepsilon}(t) \geq \frac{C_{\varepsilon}}{t^{1+\varepsilon}}, \quad t \rightarrow+\infty .
$$

Our approch is based on the theory of Riesz basis and earlier results of Littman and Markus [9] on the hybrid system.

To our knowledge, the estimate (1.3) and the optimality is new even in onedimensionel cas. In fact, there were several works on the energy decay rate for smooth solution of wave equation [7], [8]. In [8] Lebeau and Robbiano considered the boundary stabilization for wave equation. In particular, it was shown that the energy has a decay rate just like $\frac{1}{(\ln t)^{2-\delta}}, \quad \delta>0$ for any $u_{0} \in D(A)$.

Unlike the spectrum method, the multiplier method does not necessitate any knowledge of the spectrum of the system. It is simple and can be adapted to the study of other problems in any spatial dimension (see Rao [11], Rao-Wehbe [13]). But, the spectrum is essentially limited to one-dimensional problems.

\section{Rational energy decay rate by a multiplier method}

Let $u=(y, z, \eta)$ be a smooth solution of the system (1.1). We define the associated energy by:

$$
E(t)=\frac{1}{2}\left\{\int_{0}^{1}\left(y_{t}^{2}+y_{x}^{2}\right) d x+\eta^{2}\right\}
$$


Then a direct computation gives

$$
\frac{d E(t)}{d t}=-\beta \eta^{2}(t), \quad \forall t \geq 0 .
$$

In this section, using a multiplier method, we will establish the rational energy decay rate for smooth solution of the system (1.1).

Theorem 2.1 Assume that $\beta>0$. Then for any $u_{0} \in D(A)$, there exists a constant $M>0$ depending only on $u_{0}$ such that :

$$
E(t) \leq E(0) \frac{2 M}{M+t}, \quad \forall t \geq 0
$$

Proof. The idea of the proof consists in using the multiplier $x y_{x} E(t)$ which is used in Rao [11]. Let $0 \leq S<T<+\infty$, we multiply the wave equation by $2 x y_{x} E(t)$ and integrate by parts:

$$
\int_{S}^{T} E(t) d t \leq 4 E(0) E(S)+E(S) \int_{S}^{T}\left(y_{t}^{2}(1, t)+y_{x}^{2}(1, t)\right) d t
$$

On the other hand, from (2.2) we deduce that

$$
\beta \int_{S}^{T} \eta^{2}(t)=-\int_{S}^{T} E^{\prime}(t) d t \leq E(0), \quad \beta \int_{S}^{T} \eta_{t}^{2}(t) d t \leq E_{1}(0)
$$

where the energy of high order $E_{1}(t)$ is defined by

$$
E_{1}(t)=\frac{1}{2}\left\|u^{\prime}(t)\right\|_{E}^{2}, \quad \forall t \geq 0 .
$$

Combining (2.5) and (1.1) we obtain that

$$
\begin{gathered}
\int_{S}^{T}\left(y_{t}^{2}(1, t)+y_{x}^{2}(1, t)\right) d t=\int_{0}^{1}\left(\left(\eta_{t}+\beta \eta\right)^{2}+\eta^{2}\right) d t \leq \\
2\left(\frac{E_{1}(0)}{\beta E(0)}+\beta+\frac{1}{\beta}\right) E(0) E(S) .
\end{gathered}
$$

Inserting (2.6) into (2.4) gives that

$$
\int_{S}^{T} E^{2}(t) d t \leq M E(0) E(S), \quad \forall 0 \leq S \leq T<\infty
$$

where we have put:

$$
M=2\left(\frac{E_{1}(0)}{\beta E(0)}+\beta+\frac{1}{\beta}+2\right) .
$$

Thanks to a classic result of Haraux (see [5]) we deduce the rational decay rate (2.3) from the estimation (2.7). The proof is thus complete. 


\section{Rational energy decay rate by a spectrum method}

Let $u_{0} \in D(A)$, we define the optimal rational energy decay rate $\omega\left(u_{0}\right)$ by:

$$
\omega\left(u_{0}\right)=\sup \left\{\alpha \in \mathbb{R}: \quad E(t)=\frac{1}{2}\left\|S_{A+B}(t) u_{0}\right\|_{\mathcal{H}}^{2} \leq \frac{C}{t^{\alpha}}\right\} .
$$

¿From theorem 2.1, we have $\omega\left(u_{0}\right) \geq 1$ for any $u_{0} \in D(A)$. In the following, we will prove that this upper-bound is optimal in the sense that for any $\varepsilon>0$ there exists $u_{0}^{\varepsilon} \in D(A)$ such that $\omega\left(u_{0}^{\varepsilon}\right)=1+\varepsilon$.

We first recall the following result (see Littman-Markus [9]):

Lemma 3.1 Consider a $C^{0}$-semigroup $S_{\mathcal{A}}(t)$ acting on a real or complex Hilbert space $E$, with infinitesimal generator $\mathcal{A}$. Assume that:

(i) The eigenvalue $\lambda_{n}$ of $\mathcal{A}$ has the following form $\lambda_{n}=-\sigma_{n}+i \tau_{n}$ such that

$$
\sigma_{n}>\frac{a}{n^{\delta}}, \quad a>0, \delta>0 .
$$

(ii) The eigenvectors $\left\{\Phi_{n}\right\}_{n \geq 1}$ associated to the eigenvalues $\lambda_{n}$ form a Riesz basis in $\mathcal{H}$.

(iii) Let $u_{0} \in \mathcal{H}$ such that

$$
u_{0}=\sum_{n \geq 1} a_{n} \Phi_{n}, \quad\left|a_{n}\right| \leq \frac{b}{n^{q}}, b>0, q>\frac{1}{2}
$$

Then there exists a constant $C>0$ depending on $u_{0}$ such that

$$
\left\|S_{\mathcal{A}}(t) u_{0}\right\|_{\mathcal{H}} \leq \frac{C}{t^{(q-1 / 2) / \delta}}, \quad \forall t>0 .
$$

Remark 3.1 In fact if we assume that

$$
\sigma_{n} \sim \frac{1}{n^{\delta}} \quad \text { and } \quad a_{n} \sim \frac{1}{n^{q}} .
$$

Then we have

$$
\left\|S_{\mathcal{A}}(t) u_{0}\right\|_{\mathcal{H}} \sim \frac{1}{t^{(q-1 / 2) / \delta}}, \quad \forall t>0 .
$$

Let $\lambda \in \mathbb{C}$ be an eigenvalue and $u=(y, z, \eta) \in D(A)$ be the eigenvector of $A+B$

$$
(A+B) u=\lambda u \text {. }
$$

Then it follows that

$$
\begin{cases}y_{x x}-\lambda^{2} y=0, & 0<x<1, \\ y(0)=0, & \\ \lambda\left((\lambda+\beta) y_{x}(1)+\lambda y(1)\right) & =0 .\end{cases}
$$


Notice that $\lambda=0$ is not an eigenvalue of $A+B$. Hence using the bounded condition at $x=1$ we deduce that $\lambda$ is an eigenvalue of $A+B$ if and only if $\lambda$ is a zero of the function

$$
f(z)=(z+\beta+1) e^{2 z}+z+\beta-1 .
$$

Using Hadamard's factorization theorem we can prove that $f(z)$ has an infinite number of roots $\lambda_{n}$ for $n \in \mathbb{Z}$, which appear in conjugate pairs and $\left|\lambda_{n}\right|$ goes to infinity as $|n|$ goes to infinity. Moreover the algebraic multiplicity of $\lambda_{n}$ is one.

Lemma 3.2 Assume that $\beta \geq 0$. Then we have the following asymptotic expansion:

$$
\lambda_{n}=i\left(n \pi+\frac{\pi}{2}+\frac{1}{n \pi}-\frac{1}{2 n^{2} \pi}\right)-\frac{\beta}{n^{2} \pi^{2}}+O\left(\frac{1}{n^{3}}\right) .
$$

Proof. Writting (3.2) into the form:

$$
e^{2 \lambda_{n}}=(-1)\left(1-\frac{2}{\lambda_{n}+\beta+1}\right) .
$$

We obtain

$$
\lambda_{n}=\frac{1}{2} \ln (-1)+\frac{1}{2} \ln \left(1-\frac{2}{\lambda_{n}+\beta+1}\right)+i n \pi .
$$

Since $\left|\lambda_{n}\right|$ goes to infinity, we obtain the first asymptotic expansion:

$$
\lambda_{n}=i n \pi+i \frac{\pi}{2}+O\left(\frac{1}{n}\right),
$$

which implies that

$$
\ln \left(1-\frac{2}{\lambda_{n}+\beta+1}\right)=-\frac{2}{\lambda_{n}}+\frac{2 \beta}{\lambda_{n}^{2}}+O\left(\frac{1}{\lambda_{n}^{3}}\right)=i\left(\frac{2}{n \pi}-\frac{1}{n^{2} \pi^{2}}\right)-\frac{2 \beta}{n^{2} \pi^{2}}+O\left(\frac{1}{n^{3}}\right) .
$$

Then we have

$$
\ln \left(1-\frac{2}{\lambda_{n}+\beta+1}\right)=i\left(\frac{2}{n \pi}-\frac{1}{n^{2} \pi^{2}}\right)-\frac{2 \beta}{n^{2} \pi^{2}}+O\left(\frac{1}{n^{3}}\right) .
$$

Inserting (3.7) into (3.5), we obtain the asymptotic expansion (3.3). This achieves the proof. In particular we will numerote the eigenvalues $\lambda_{n}$ of high frequencies following the asymptotic form (3.3).

Now let

$$
\left.D_{n}=\right]-n \pi, n \pi[\times]-n \pi, n \pi[\text {. }
$$

Lemma 3.3 There exists an integer $N$ such that for $n>N$ the function $f(z)$ has $2 n+1$ roots in $D_{n}$.

Proof. We first prove that $f(z)$ and $g(z)=(z+1) e^{2 z}+z-1$ have the same number of roots in $D_{n}$. By virtue of Rouché's theorem, it is sufficient to prove the following estimate

$$
|f(z)-g(z)|<|g(z)|, \quad \forall z \in \partial D_{n} .
$$


Let $z= \pm i n \pi+x, \quad x \in[-n \pi, n \pi]$, then we have :

$$
1+e^{2 z}=1+e^{2 x} \cdot e^{2 i n \pi}=1+e^{2 x}>1 .
$$

Let $z= \pm n \pi+i y, \quad y \in[-n \pi, n \pi]$, then we have :

$$
\left|1+e^{2 z}\right| \geq\left|1-e^{2 n \pi}\right|>1 / 2, \quad \forall n \geq 1 .
$$

On the other hand, since $|z+1| \geq n \pi-1$, then we obtain

$$
|f(z)-g(z)|=\beta\left|1+e^{2 z}\right|<|z+1|\left|1+e^{2 z}\right|-2<|g(z)|, \quad \forall z \in \partial D_{n},
$$

provided that $n>\frac{\beta+5}{\pi}$.

We next determine the number of the zeros of $g(z)$ in the domain $D_{n}$. Let $i \mu_{n} \in$ $i \mathbb{R}$ be a zero of $g(z)$, then we have $\tan (\mu)+\mu=0$ which admits a unique zero in each interval $\left[\left(k-\frac{1}{2}\right) \pi, k \pi\right], 1 \leq k \leq n$. Since $\mu_{0}=0$ is obviously a zero and since $\tan \mu+\mu$ is odd, we obtain exactly $2 n+1$ zeros in $D_{n}$.

Let $\widetilde{\lambda}_{n}=i \mu_{n} \in i \mathbb{R}$ be an eigenvalue of the skew adjoint operator $A$ and $\widetilde{\Phi}_{n}=$ $\left(\widetilde{y}_{n}, \widetilde{z}_{n}, \widetilde{\eta}_{n}\right)$ be the associated eigenvector:

$$
\widetilde{\Phi}_{0}=\left(\begin{array}{c}
x \\
0 \\
-1
\end{array}\right) ; \quad \widetilde{\Phi}_{n}=\left(\begin{array}{c}
\frac{1}{\widetilde{\lambda}_{n}} \sinh \left(\widetilde{\lambda}_{n} x\right) \\
\sinh \left(\widetilde{\lambda}_{n} x\right) \\
-\cosh \left(\widetilde{\lambda}_{n}\right)
\end{array}\right), \quad \forall n \in \mathbb{Z}^{*} .
$$

Since $A$ is skew adjoint with compact resolvent, then the system of eiganvectors is complete in $\mathcal{H}$ On the other hand, a directe calculation gives:

$$
\left\|\widetilde{\Phi}_{n}\right\|_{\mathcal{H}}^{2}=\left|\cos \left(\mu_{n}\right)\right|^{2}+1 \rightarrow 1, \text { as } n \rightarrow+\infty .
$$

It follows that $\left(\widetilde{\Phi}_{n}\right)_{n \in \mathbb{Z}}$ is a Riesz basis of $\mathcal{H}$.

Now let $\lambda_{n}$ be an eigenvalue of $A+B$, and $\Phi_{n}=\left(y_{n}, z_{n}, \eta_{n}\right)$ be the associated eigenvector:

$$
\Phi_{n}=\left(\begin{array}{c}
\frac{1}{\lambda_{n}} \sinh \left(\lambda_{n} x\right) \\
\sinh \left(\lambda_{n} x\right) \\
-\cosh \left(\lambda_{n}\right)
\end{array}\right), \quad \forall n \in \mathbb{Z} .
$$

Theorem 3.1 Assume that $\beta>0$. Then the system of root vectors $\left(\Phi_{n}\right)_{n \in \mathbb{Z}}$ is a Riesz basis in $\mathcal{H}$.

Proof. Using Lemma 3.2 we deduce that there exists an integer $N$ such that for any $n>N$ we have $\lambda_{n}=-\sigma_{n}+\widetilde{\lambda}_{n}$ where

$$
\tilde{\lambda}_{n}=i\left(n \pi+\frac{\pi}{2}+\frac{1}{n \pi}-\frac{1}{n^{2} \pi}+O\left(\frac{1}{n^{3}}\right)\right), \quad \sigma_{n}=\frac{\beta}{n^{2} \pi^{2}}+O\left(\frac{1}{n^{3}}\right) .
$$

Then we deduce that

$$
\left|y_{n x}(x)-\widetilde{y}_{n x}(x)\right|=\left|\cosh \left(\lambda_{n} x\right)-\cosh \left(\widetilde{\lambda}_{n} x\right)\right| \leq C_{0}\left|\lambda_{n}-\widetilde{\lambda}_{n}\right| \leq \frac{C}{n^{2}},
$$




$$
\left|z_{n}(x)-\widetilde{z}_{n}(x)\right|=\left|\sinh \left(\lambda_{n} x\right)-\sinh \left(\widetilde{\lambda}_{n} x\right)\right| \leq C_{1}\left|\lambda_{n}-\widetilde{\lambda}_{n}\right| \leq \frac{C}{n^{2}}
$$

and

$$
\left|\eta_{n}-\widetilde{\eta}_{n}\right|=\left|\cosh \left(\lambda_{n}\right)-\cosh \left(\widetilde{\lambda}_{n}\right)\right| \leq C_{2}\left|\lambda_{n}-\widetilde{\lambda}_{n}\right| \leq \frac{C}{n^{2}}
$$

Combing (3.13), (3.14) and (3.15) we get

$$
\sum_{n \in \mathbb{Z}}\left\|\widetilde{\Phi}_{n}-\Phi_{n}\right\|_{\mathcal{H}}^{2}<+\infty
$$

Since the system $\left(\Phi_{n}\right)_{n \in \mathbb{Z}}$ is $\omega$-linearly independent (see Lemma A.6 in [3]) and quadratically close to the Riesz basis $\left(\widetilde{\Phi}_{n}\right)_{n \in \mathbb{Z}}$, applying Bari's theorem we conclude that $\left(\Phi_{n}\right)_{n \in \mathbb{Z}}$ is also a Riesz basis in $\mathcal{H}$. This achieves the proof.

Theorem 3.2 Assume $\beta>0$. Then we have

$$
\inf _{u_{0} \in D(A)} \omega\left(u_{0}\right)=1
$$

Proof. Let $\varepsilon>0$, we define $u_{0}^{\varepsilon}$ by

$$
u_{0}^{\varepsilon}=\sum_{n \in \mathbb{Z}^{*}} \frac{1}{n^{3 / 2+\varepsilon}} \Phi_{n}
$$

Then we have

$$
(A+B) u_{0}^{\varepsilon}=\sum_{n \in Z^{*}} \frac{\lambda_{n}}{n^{3 / 2+\varepsilon}} \Phi_{n}
$$

Using the Lemma 3.2 we deduce that $\left|\frac{\lambda_{n}}{n^{3 / 2+\varepsilon}}\right| \sim \frac{1}{n^{1 / 2+\varepsilon}}$. Since $\left(\Phi_{n}\right)_{n \in \mathbb{Z}}$ is a RiesZ basis in $\mathcal{H}$, then we have :

$$
\left\|(A+B) u_{0}^{\varepsilon}\right\|_{\mathcal{H}}^{2} \sim \sum_{n \in \mathbb{Z}^{*}}\left|\frac{\lambda_{n}}{n^{3 / 2+\varepsilon}}\right|^{2} \sim \sum_{n \in \mathbb{Z}^{*}} \frac{C}{n^{1+2 \varepsilon}}<\infty .
$$

This implies that $u_{0}^{\varepsilon} \in D(A)$. Thanks to Lemma 3.1 and Remark 3.1 (with $q=$ $3 / 2+\varepsilon$ and $\delta=2$ ) we deduce that

$$
E_{\varepsilon}(t)=\frac{1}{2}\left\|S_{A+B}(t) u_{0}^{\varepsilon}\right\|_{\mathcal{H}}^{2} \sim \frac{C_{\varepsilon}}{t^{1+\varepsilon}}, \quad \forall t>0
$$

where $C_{\varepsilon}$ is a constant depending on $u_{0}^{\varepsilon}$. It follows that:

$$
\omega\left(u_{0}^{\varepsilon}\right)=1+\varepsilon .
$$

On the other hand, from Theorem 2.1 we deduce that

$$
\omega\left(u_{0}\right) \geq 1, \text { for any } u_{0} \in D(A) .
$$

Then we deduce that

$$
1 \leq \inf \omega\left(u_{0}\right) \leq 1+\varepsilon .
$$

Since $\varepsilon$ can be arbitrarily small, we obtain (3.16). This achieves the proof.

\section{Acknowledgements}

The author would like to thank professor Bopeng Rao for very helpful discussions and suggestions. 


\section{References}

[1] C.D. Benchimol, A note on weak stabilizability of contraction semigroups, SIAM J. Control Optim. 16 (1978), 373-379.

[2] H. Brezis, Analyse Fonctionelle, Théorie et Applications, Masson, Paris, 1992.

[3] F. Conrad and Ö. Morgül, On the stabilization of flexible beam with a tip mass, SIAM J. Control Optim. Vol. 36, No. 6, pp. 1962-1986, 1998.

[4] B.A. Francis, $H_{\infty}-$ control theory, Lecture Notes in Control and Sciences (1986).

[5] V. Komornik, Exact Controllability and Stabilization, Masson, Paris, 1994.

[6] V. Komornik and B. Rao, Boundary stabilization of compactly coupled wave equations, Asymptotic Analysis, 14, (1997) 339-359.

[7] G. Lebeau Équation des ondes amorties, Kluwer Acad. Publ., Dordrecht, 1996.

[8] G. Lebeau and L. Robbiano, Stabilization of the wave equation by the boundary, Progr. Nonlinear Differential Equations Appl., 21, Birkhäuser Boston, Boston, MA, 1996.

[9] W. Littman and L. Markus, Some recent results on control and stabilization of flexible structures, Proc. COMCON Workshop, Montpellier 1987.

[10] A. Pazy, Semigroups of Linear Operators and Applications to Partial Differential Equations, Applied Mathematical Sciences, V. 44, Springer-Verlag, 1983.

[11] B. Rao Stabilization of elastic plates with dynamical boundary control, SIAM J. Control Optim. Vol. 36, No. 1, pp. 148-163, 1998.

[12] B. Rao, A compact perturbation method for the boundary stabilization of the Rayleigh beam equation, Appl. Math. Optim., 33 (1996), pp. 253-263.

[13] B. Rao et A. Wehbe, Stabilization frontière de plaques de Kirchhoff avec résolvente non-compacte, C. R. Acad. Sci. Paris, t. 328, Série 1, P. 591-596, 1999.

[14] D.L. Russell, Decay rates for weakly damped systems in Hilbert space obtained with control-theoretic methods, J. Differential Equations, 19 (1975), 344-370.

[15] D.L. Russell, A General framework for the study of indirect damping mechanisms in elastic systems, J. Math. Anal and Appl. (1993). 